\title{
ES NECESARIO REVISAR EL PROYECTO DE LA LÍNEA 2 DEL TREN ELÉCTRICO DE LIMA
}

\author{
It is necessary to assess 'Linea 2'Electric Train of Lima Project
}

Arturo Velásquez Jara ${ }^{1}$

PRESENTACIÓN: 21 DE NOVIEMBRE DE 2018

\section{RESUMEN}

En el artículo se revisa el caso del proyecto Línea 2 del Metro de Lima que, con una extensión de $35 \mathrm{~km}$, conectará el distrito de Ate, en el Este de la ciudad, con el Callao, y que incluirá un ramal de $8 \mathrm{~km}$ a lo largo de la avenida Elmer Faucett para conectar dicha línea con el aeropuerto Jorge Chávez. El proyecto fue entregado en concesión al Consorcio Nuevo Metro de Lima, integrado por firmas peruana, española e italiana, en el año 2014. Comprometía una inversión total de $\$ 6,620$ millones, incluido el cofinanciamiento del Estado por $\$ 3,695$ millones.

Las fases iniciales del proyecto, desde los estudios de preinversión y luego el concurso para selección del concesionario, se realizaron de manera tal que se las puede calificar como apresuradas, lo que llevó a decisiones que han ocasionado que el proyecto no se desarrolle en forma eficiente. En el caso de la etapa de preinversión, se ha revisado el estudio de factibilidad del proyecto y se ha encontrado que los análisis técnicos y económicos son relativamente superficiales, algo que contrasta con la importancia del proyecto. Las principales deficiencias encontradas incluyen: insuficiente análisis de alternativas tecnológicas, pésima formulación del programa de ejecución del proyecto y débil análisis de evaluación del proyecto.

Habiéndose iniciado las obras en diciembre del 2014, a setiembre del 2018 se ha anunciado que el avance del proyecto es de solamente $24 \%$, lo que evidencia un exagerado retraso en su ejecución, considerando que la culminación total del proyecto estaba programada para el año 2019.

Además de múltiples complicaciones técnicas, administrativas y legales que han afectado el desarrollo
APROBACIÓN: 30 DE NOVIEMBRE DE 2018

1 Profesor Principal en la Universidad Ricardo Palma y de la Universidad Nacional de Ingeniería, con estudios en Ingeniería Civil y de segunda especialización en Proyectos de Inversión. Maestría en Gerencia de la Construcción Moderna, Doctorado en Medio Ambiente y Desarrollo Sostenible. E-mail: avelasquezjara@yahoo.es 
del plan, el concesionario ha presentado un recurso de arbitraje ante la CIADI (entidad del Banco Mundial) argumentando que los terrenos separados para la ejecución de las obras no cumplen las condiciones pactadas en el contrato, lo que les ha ocasionado un retraso de más de 1 año. Por ello, solicitan una compensación de 260 millones de dólares.

Se considera que estamos a tiempo de una revisión radical del proyecto para modificarlo a una solución elevada (viaducto) a partir de la plaza Bolognesi y en todo su desarrollo en la provincia del Callao, lo que significaría una reducción en los costos de inversión del orden de $\$ 1,500$ millones. Para esto, se propone se contrate a una consultora con reconocida experiencia y con participación de entidades internacionales de prestigio como el Banco Mundial, la que analizaría exhaustivamente el caso para encontrar una buena solución para este cambio en la opción tecnológica considerando sus implicancias en los aspectos financieros y legales del contrato, en resguardo del interés público.

Palabras clave: Concesión de proyectos, estudios de factibilidad, costos de inversión, rentabilidad social. that the separate lands for the execution of the works do not meet the conditions agreed in the contract, which has caused them a delay of more than 1 year, for which they request compensation of 260 million dollars.

It is considered that we are in time for a radical revision of the project to modify it to a high solution (viaduct) from Plaza Bolognesi and throughout its development in the province of Callao, which would mean a reduction in investment costs of the order of $\$ 1,500$ million. For this purpose it is proposed to hire a consultant with recognized experience and with participation of prestigious international entities such as the World Bank, which would thoroughly analyze the case to find a good solution for this change in the technological option considering its implications in the financial and legal aspects of the contract, in defense of the public interest.

Keywords: Project concession, Feasibility studies, Investment costs, Social profitability

\section{DESARROLLO DEL TEMA ANTECEDENTES}

Dentro de las propuestas de solución a la problemática del transporte en ciudades como Lima, se viene implementando una red de trenes urbanos (metros) como solución tecnológica que se aplica en las grandes ciudades del mundo, dado que atienden significativas demandas de transporte en condiciones de alta calidad, aunque requieren importantes inversiones para su realización.

Después de años dedicados a diversos estudios, análisis de alternativas y de, a veces, precipitadas y discutibles decisiones políticas, por fin Lima pudo contar con una primera línea de metro, la denominada Línea 1, cuya primera etapa entre Villa El Salvador y el Centro de Lima, fue parcialmente construida entre los años 1986 a 1990, aunque sin entrar en funcionamiento por un buen número de años al no haberse completado la infraestructura requerida ni contar con el equipo rodante y otras complicaciones propias de estos grandes proyectos. Luego de estar paralizadas por muchos años, las obras de infraestructura de esta Línea 1 se reiniciaron el ańo 2010 y se culminaron el 2011, y entraron en funcionamiento comercial en el año 2012. La segunda etapa de esta línea, del centro de Lima a San Juan de Lurigancho, inició su construcción el 2012 y entró en funcionamiento en el año 2014.

La segunda Línea del sistema de trenes eléctricos de Lima (Línea 2), que se encuentra en actual proceso de construcción, se extiende entre los distritos de Ate y Callao. Se ha incluido, además, en el contrato de concesión, un tramo de la Línea 4, para conectar la Línea 2 con el Aeropuerto Jorge Chávez. La buena pro de la concesión fue otorgada en marzo de 2014 al Consorcio Nuevo Metro de Lima, integrado por Cosapi S.A. (Perú), Impregilo S.p.A. (Italia), Iridium Concesiones de Infraestructura S.A. (España), Vialia Sociedad Gestora de Concesiones de Infraestructura S.L. (España), Ansaldo Breda S.p.A. (Italia) y Ansaldo STS S.p.A. (Italia).

El consorcio ganador pidió en su propuesta económica un cofinanciamiento de 3,695 millones de dólares, para una inversión total de 6,620 millones de dólares, y también pidió 108 millones de dólares por Retribución de Operación y Mantenimiento, habiéndose dado la concesión por un plazo de 35 años. Este proyecto ha sido bastante cuestionado, dada la magnitud de la inversión involucrada (6\% del PBI anual) que lo hace uno de los proyectos más caros de su tipo en el mundo. En el presente 
artículo se abordan los aspectos técnico-económicos y de gestión del proyecto, y se proponen algunas recomendaciones para mejorar las condiciones en que podría manejarse, para un mejor resguardo de los recursos públicos, considerando que la magnitud de las inversiones involucradas representa un significativo esfuerzo económico por parte del Estado.

\section{LA APROBACIÓN DEL PROYECTO LÍNEA 2 Y RAMAL DE LÍNEA 4 DEL METRO DE LIMA: DECISIONES INEFICIENTES}

Este proyecto, como propuesta genérica, ya figuraba en los planes metropolitanos desde los ańos 80 y fue formalmente incluido en los planes de inversión el año 2010 mediante el Decreto Supremo N ${ }^{\circ}$ 059-2010-MTC que definió la Red Básica del Metro de Lima. Luego, mediante Decretos de Urgencia 001 y 002-2011, fue declarado de necesidad nacional y de ejecución prioritaria. El proyecto contó con estudio de preinversión a nivel de perfil desarrollado el año 2012 y, posteriormente, se formuló el estudio de factibilidad [1] que fue terminado en julio de 2013. Ambos estudios fueron realizados por el consorcio Geodata-Esan-Serconsult. La declaratoria de viabilidad fue dada por el Ministerio de Economía y Finanzas y el Ministerio de Transportes en agosto de 2013.

Se hace notar que la convocatoria al concurso público para selección del concesionario fue lanzada por ProInversión en enero del 2013, esto es sin contarse todavía con el informe de viabilidad. Al respecto, se debe señalar que la normatividad vigente en ese momento ${ }^{2}$ no establecía claramente este requisito como debería corresponder al tratarse de inversiones en que se involucran recursos públicos.

La premura por iniciar este proyecto podría explicarse por la necesidad de buscar soluciones al creciente problema de transporte y congestionamiento vehicular que padece la ciudad de Lima. Sin embargo, además de la crítica formal por lanzar la convocatoria internacional de un proyecto cuando no se contaba con el estudio de viabilidad que debe tener toda inversión pública, en este trabajo también se tiene que cuestionar la etapa de preinversión que, aunque fue realizada por consultoras de solvencia reconocida, tienen graves deficiencias que un análisis más objetivo no deja de evidenciar conforme se indica a continuación:

\section{A) insuficiente análisis de alternativas tecnológicas}

Una primera deficiencia o grave omisión es que en el estudio de factibilidad realizado no se presenta el análisis de alternativas tecnológicas que sustente por qué el proyecto contratado considera que los 35 km de longitud de la Línea 2, incluido el ramal por la avenida Faucett, se construirán totalmente en forma subterránea a más de $30 \mathrm{~m}$ bajo la superficie. Esta decisión sin fundamento técnico-económico aumenta significativamente los costos, porque hace necesario construir túneles, pasajes, escaleras mecánicas y otras estructuras subterráneas, además de las estaciones de embarque y desembarque de pasajeros, que requieren también elevadas sumas de dinero, lo que supone, a su vez, elevados costos de operación futuros, consecuencia del funcionamiento de una gran cantidad de escaleras eléctricas y elevadores.

Como referencia general, se estima que los costos para construir $1 \mathrm{~km}$ de vía subterránea para los trenes urbanos ascienden a 2 o 3 veces el costo de construir la vía del tren en una alternativa de viaducto (como es el caso de la Línea 1 en actual funcionamiento). Por tanto, era muy importante estudiar cuidadosamente si se justificaba que todos los $35 \mathrm{~km}$ fueran ejecutados en la opción subterránea. En

2 Texto Único Ordenado de las normas con rango de Ley que regulan la entrega en concesión al sector privado de las obras públicas de infraestructura y de servicios públicos. D. S. No 059-96-PCM. 
todo caso, podría limitarse esta opción para el recorrido en la zona céntrica de la ciudad y proyectar el resto del recorrido, desde Breña hasta el Callao, e inclusive el ramal de la Línea 4, en forma de viaducto, ahorrando casi 1,500 millones de dólares en la inversión requerida.

La decisión de desarrollar todo el proyecto en forma subterránea ha sido criticada tanto por ingenieros como por economistas [2]. Frente a tales cuestionamientos, autoridades comprometidas con estas decisiones han manifestado que se decidió que todo el tren fuera subterráneo para no tener problemas con las expropiaciones. Al respecto, debe hacerse notar que necesariamente una obra de esta magnitud requiere afrontar significativas expropiaciones, cualquiera que sea la opción tecnológica tomada, ya sea la alternativa subterránea o de viaducto. Además, este argumento es totalmente objetable si se tiene en cuenta que, iniciadas ya las obras del proyecto diseñado en subterráneo, se han presentado paralizaciones por problemas de expropiaciones por varios meses. Debe comentarse que, lamentablemente, la incapacidad para realizar expropiaciones de manera expeditiva es una evidencia de la baja capacidad de gestión de los responsables de este importante proyecto, incapaces de culminar este tipo de procesos, que siempre tardan mucho más de lo esperado.

La posibilidad de modificar la decisión de realizar el proyecto todo en subterráneo fue planteada, incluso, por otros dos consorcios postores al concurso para concesión: el Consorcio Metro Subterráneo de Lima, formado por Astaldi S.P.A. (Italia) y Controladora de Operaciones de Infraestructura S.A. de C.V. (México), y Metro de Lima 2, compuesto por Odebrecht LatInvest Perú S.A.C. (Brasil), Graña y Montero S.A.A. (Perú), Constructora Andrade Gutiérrez S.A. Sucursal del Perú (Brasil) y Constructora Queiroz Galvao S.A. Sucursal Perú (Brasil). Los dos consorcios, al no recibir respuesta positiva a su propuesta, no presentaron su oferta económica, de manera que en marzo de 2014 fue otorgada la buena pro de la concesión a postor único, lo que provocó diversas manifestaciones críticas, inclusive del Colegio de Ingenieros [3].

\section{b) Pésima formulación del Programa de ejecución del proyecto}

Otra lamentable debilidad de los estudios de preinversión de este proyecto se aprecia al revisar el estudio de factibilidad realizado en el año 2013, en el cual los consultores, con un optimismo digno de mejor causa, consideraron que la primera etapa de esta línea ya estaría en operación en el 2018, lo que evidencia también su carencia de realismo al momento de formular el programa general de desarrollo del proyecto. El referido optimismo ha sido lamentablemente contradicho por la realidad.

Desde sus inicios, en diciembre del 2014, las obras han venido sufriendo múltiples retrasos en su ejecución, de tal manera que en la evaluación efectuada a octubre del 2016 se apreció que se habían demorado 2 años para construir 2 estaciones, y en noviembre del 2016, luego de 2 ańos de contrato, solo se habían avanzado $2 \mathrm{~km}$ de la longitud total de $35 \mathrm{~km}$. Se aprecia que, desde el inicio, la ejecución del proyecto ha afrontado múltiples dificultades, algunas debido a la lentitud de las entidades públicas. Hay que considerar, por ejemplo, la necesidad de coordinación con la Municipalidad de Lima para conseguir autorizaciones sobre la compatibilidad vial del proyecto con la zona urbana, a lo cual se atribuyó parte del retraso en el inicio de las obras. Otra causa fue la demora en la aprobación de los estudios definitivos del primer tramo por parte del ente supervisor, el cual, además, fue designado de manera retrasada en marzo de 2015.

Otra evidencia de la deficiente programación general del proyecto se observa cuando, ya en setiembre de 2016, llegaron los primeros trenes de Italia, que continuarán guardados por lo menos 6 años hasta que por fin puedan entrar en funcionamiento. Las tuneladoras también llegaron en marzo de 2016 y se espera que entren propiamente en funcionamiento a fines del próximo año, ya que previamente debe concluirse la estación San Juan de Dios y, además, debe contarse con un número importante de dovelas para el túnel cuya producción recién se iniciará a mediados del 2019 [4]. Tanto las 
tuneladoras como los trenes son inversiones importantes que, al haberse realizado tan anticipadamente, significan un alto costo financiero que podría haberse evitado con una programación más realista.

\section{c) Débil análisis de evaluación del proyecto}

Otra deficiencia muy grave en el estudio de factibilidad se relaciona con la forma en que se han calculado los indicadores de evaluación social, y cuáles son el Valor Actual Neto y la Tasa Interna de Retorno, ya que la cuantificación de los beneficios y costos proyectados a precios sociales reposan en supuestos bastante discutibles, como se explica a continuación.

Un primer supuesto, muy cuestionable, consideró importantes beneficios sociales por revalorización de inmuebles en las zonas cercanas a las estaciones por un monto de 1,921 millones de dólares y que se otorgarían en el año 2014. Bastaría que estos beneficios se redujeran en $40 \%$ para que el Valor Actual Neto (VAN) del proyecto, que según el estudio arrojó 759 millones de dólares, a una tasa de descuento de $9 \%$, pase a ser negativo, lo que significa que el proyecto no ofrecería una rentabilidad social aceptable.

Además, el estudio consideró que el costo de inversión por expropiaciones solo llegaría a 107 millones de dólares, estimación que ha quedado ampliamente superada por la realidad, pues cálculos más realistas posteriores elevan esta cifra a más 600 millones de dólares. Este incremento en los costos, del orden de 500 millones de dólares, haría que la rentabilidad negativa del proyecto se agrave, haciendo ver lo muy cuestionable que fue la decisión de llevar adelante el proyecto con las características planteadas.

De hecho, si se disminuyen los supuestos beneficios por incremento en el valor de los inmuebles como consecuencia de la existencia del proyecto, considerando solamente los beneficios por ahorros de tiempo de los usuarios, por ahorro en costos de operación vehicular y otros de menor cuantía, y se agregan 500 millones a los costos de expropiaciones, se obtiene un Valor Actual Neto negativo de -1,662 millones de dólares, lo que indica que los beneficios del proyecto serían muy insuficientes para justificar su altísimos costos, implicando que el proyecto tal como está planteado ocasionará una gran pérdida para la economía del país. En ese sentido, debió minimizarse la inversión evitando en lo posible la opción de todo subterráneo.

\section{LA SITUACIÓN ACTUAL DE LA CONCESIÓN}

Además del fuerte retraso en el programa de ejecución ya comentado, para complicar aún más la situación de este proyecto, el concesionario ha presentado un recurso de arbitraje ante la CIADI (entidad del Banco Mundial) argumentando que los terrenos separados para la ejecución de las obras no cumplen las condiciones pactadas en el contrato, lo que les ha ocasionado un retraso de más de 1 año, por lo que solicitan una compensación de 260 millones de dólares [5].

También ha complicado el desarrollo del proyecto las discrepancias entre la concesionaria y el Consorcio Supervisor Internacional Línea 2, entidad supervisora, con relación a los aspectos técnicos sobre la resistencia a sismos de las estructuras subterráneas.

En octubre del 2016, el entonces Ministro de Transportes manifestó que el concesionario propuso modificar la parte de la Línea 2 y el tramo de la Línea 4 para hacerlo en superficie a fin de disminuir los costos de inversión. Posteriormente, se anunció que se mantendría la solución en subterráneo para todos los $35 \mathrm{~km}$ del proyecto. Esta propuesta, totalmente razonable desde el punto de vista técnicoeconómico, no habría sido aceptada dadas las implicancias legales y financieras que llevarían a una adenda al contrato de concesión, lo que aparentemente sería muy impopular. 
A setiembre del 2018, se ha anunciado que el avance del proyecto es de solamente el $24 \%$, lo que evidencia un exagerado retraso en su ejecución, considerando que la culminación total del proyecto estaba inicialmente programada para el año 2019.

\section{¿QUÉ HACER CON EL PROYECTO?}

Consideramos que estamos a tiempo de hacer una revisión radical del proyecto para modificarlo a una solución elevada (viaducto) a partir de la plaza Bolognesi, y en todo su desarrollo en la provincia del Callao. Esto permitirá disminuir apreciablemente las inversiones del proyecto, y convertirlo en uno con rentabilidad económico-social. Es necesario considerar que parte de la inversión ya realizada corresponde a las tuneladoras y al material rodante, los cuales siempre serán de utilidad en el futuro. Sería conveniente contratar a una consultora con reconocida experiencia en este tipo de proyectos, con la participación de entidades internacionales de prestigio como el Banco Mundial, que analizaría exhaustivamente el caso para encontrar una buena solución para este cambio en la opción tecnológica, considerando sus implicancias en los aspectos financieros y legales del contrato en resguardo del interés público.

El importante ahorro en los costos en el proyecto justificaría asumir la compleja y delicada decisión política de seguir las recomendaciones del referido estudio y llevar a una revisión del contrato. Esto parece que genera gran temor en las autoridades responsables del mismo, dado que el concurso fue convocado en la modalidad de "concurso de proyectos integrales", con un contrato muy complicado cuya modificación parece una decisión de alto riesgo muy difícil de asumir por los representantes del interés público, debido a la gran sensibilización y suspicacia general generados por la nefasta experiencia de la famosa adenda del aeropuerto de Chincheros.

Para que las autoridades responsables del proyecto modifiquen la resignada actitud de continuar con la costosa nativa subterránea, decidiéndose a asumir el esfuerzo de modificar el contrato, logrando la obvia reducción de los costos de inversión y la modificación de las condiciones de la concesión, se requerirá una alta dosis de valentía y, sobre todo, mucha pericia y solvencia técnica y de gestión a fin de lograr que el proyecto tenga una solución más racional eliminando las fuertes e innecesarias inversiones, lo que significaría ahorros de más de 1,500 millones de dólares. Todo esto supondría una actitud histórica: un logro emblemático de la técnica y la racionalidad sobre la lamentable irresponsabilidad con respecto al resguardo del interés público.

\section{REFERENCIAS}

[1] ProInversión. Estudio de preinversión a nivel de factibilidad de la Linea 2 y tramo de la Linea 4 del Metro de Lima. Volumen 1-Resumen ejecutivo. [En línea]. Disponible en https://www.proyectosapp.pe/RepositorioAPS/0/2/JER/LINEA2_TREN_ESTUDIOS/A9_Factibilidad_ Viable/A_9_1_vol_I_RE.pdf [Accedido: 12-jul-2018]

[2] "Declaraciones del expresidente P. P. Kuczynski", en RPP Noticias. [En línea]. Disponible en https://rpp.pe/economia/economia/kuczynski-plantea-que-linea-2-del-metro-sea-aerea-yno-subterránea-noticia-669861 [Accedido: 03-jun-2018]

[3] "Metro de Lima: Críticas por un solo postor para la Línea 2", en Perú21. [En línea]. Disponible en: https://peru21.pe/opinion/metro-lima-criticas-postor-linea-2-150945 [Accedido: 27-jun-2018]

[4] "Declaraciones del director ejecutivo del AATE, Carlos Ugaz", en diario Gestión, 07-jul-2018.

[5] Información del diario El Comercio, 04-feb-2017 


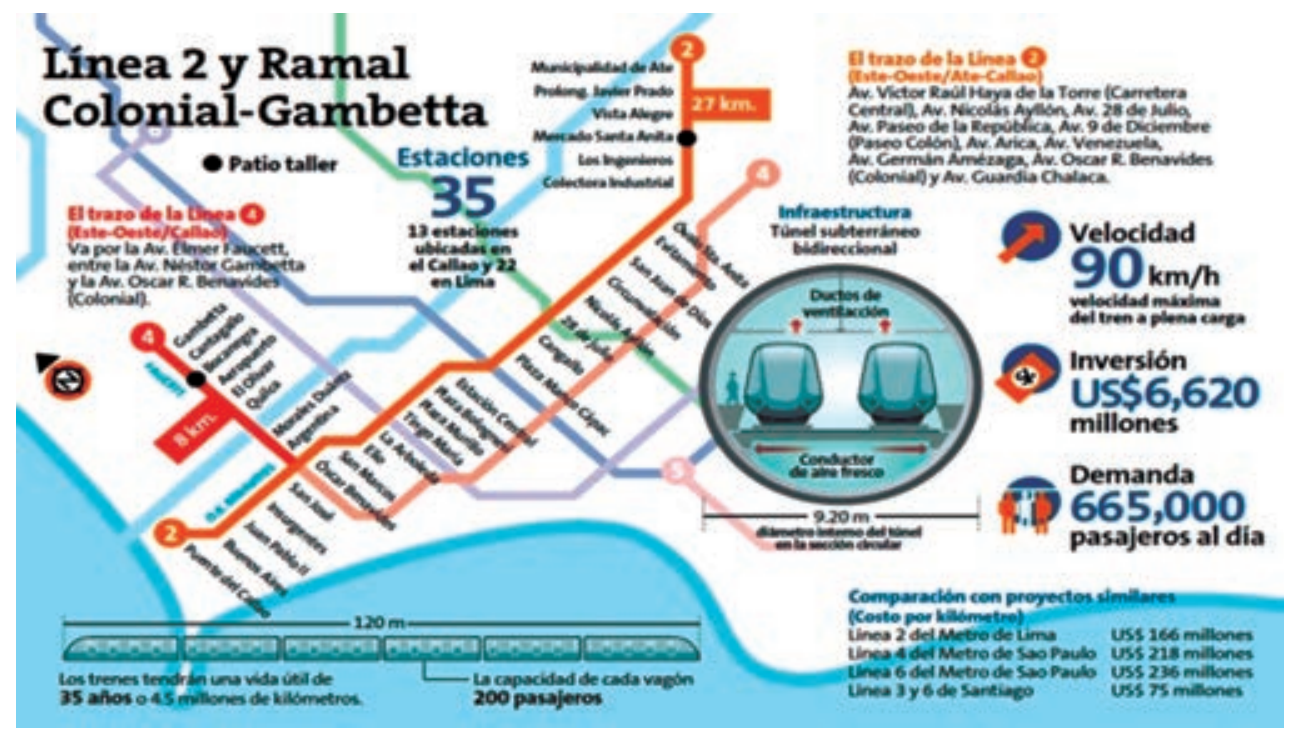

Fuente: Perú21 